\title{
Expression of Epstein-Barr nuclear antigen 1 in gastric carcinoma cells is associated with enhanced tumorigenicity and reduced cisplatin sensitivity
}

\author{
TSE-CHOU CHENG ${ }^{1 *}$, SHAN-SHAN HSIEH ${ }^{2 *}$, WEN-LIN HSU ${ }^{3}$, \\ YU-FANG CHEN ${ }^{2}$, HWEI-HON HO ${ }^{2}$ and LAI-FA SHEU ${ }^{4}$ \\ ${ }^{1}$ Departments of Surgery, Chi-Mei Medical Center, Liu-Ying Campus, Tainan; ${ }^{2}$ Department of Pathology, \\ Tri-Service General Hospital, National Defense Medical Center, Taipei; Departments of ${ }^{3}$ Radiation Oncology \\ and ${ }^{4}$ Pathology, Tzu Chi Medical Center, Taichung Campus, Taichung, Taiwan, R.O.C.
}

Received June 30, 2009; Accepted September 3, 2009

DOI: 10.3892/ijo_00000486

\begin{abstract}
Epstein-Barr nuclear antigen 1 (EBNA-1) is consistently expressed in all EBV-associated gastric carcinomas. We explored its biological effects in gastric carcinoma cells by expressing the protein in two Epstein-Barr virus (EBV)negative gastric carcinoma cell lines (SCM1 and TMC1). EBNA1-expressing SCM1 and TMC1 cells displayed no significant differences in growth rates, respectively, compared to those of vector-transfected SCM1 and TMC1 cells in vitro. However, EBNA1 was able to enhance tumorigenicity, the growth rate and the malignant histopathological grade in a xenograft nude mice test. We also evaluated whether EBNA1 caused EBNA1-expressing cells to have enhanced tumorigenicity in an immunocompetent host. We showed that EBNA1-expressing LL/2 cells (derived from lung carcinoma of a Swiss mouse) had enhanced tumorigenicity and growth ability in the immunocompetent allograft Balb/c mice test. These results support the expression of EBNA1 in EBVassociated gastric carcinoma being able to provide advantages of EBV-mediated cell growth and transformation, and to enhance the malignant potential in vivo. In a clonogenic assay, we showed that EBNA1 could reduce the sensitivity of
\end{abstract}

Correspondence to: Dr Lai-Fa Sheu, Department of Pathology, Tzu Chi Medical Center, Taichung Campus, 66 Fongsing Rd., Sec. 1, Tanzih Township, Taichung County 427, Taiwan, R.O.C.

E-mail: laifakimo@yahoo.com.tw

${ }^{*}$ Contributed equally

Abbreviations: EBNA1, Epstein-Barr nuclear antigen 1; USP7, ubiquitin-specific protease 7; LELC, lymphoepithelioma-like carcinoma; LMPs, latent membrane proteins; GAr, glycine-glycinealanine repeat sequence

Key words: Epstein-Barr nuclear antigen 1, EBV-associated gastric carcinoma, tumorigenesis, cisplatin gastric carcinoma cells (SCM1 cells) harboring wild-type p53 to cisplatin, but this was not found in mutant p53-bearing TMC1 cells. In addition, we demonstrated that EBNA1expressing SCM1 cells, but not EBNA1-expressing TMC1 cells, were associated with reduced expression levels of p53. These findings are compatible with EBNA1 efficiently competing with $\mathrm{p} 53$ for binding to ubiquitin-specific protease 7 , which causes p53 to degrade by the ubiquitin/proteasome system. These findings suggest that EBNA1 expression is able to reduce the p53 protein level, resulting in the inhibition of its functional activities. Finally, our results suggest that EBV infection with EBNA1 expression in gastric carcinomas provides advantages for host cell survival, growth ability and transformation potential involving escape from immunosurveillance and a reduction in the sensitivity to DNA damage or other apoptotic stress stimuli mediated by suppression of the wild-type p53 protein level; these are distinct from the pathogenesis of EBV-negative gastric carcinomas.

\section{Introduction}

Gastric carcinoma is a common cancer and results in about 876,000 new cases per year making it one of the leading causes of cancer deaths worldwide (1). Among gastric carcinomas, $2-16 \%$ (mean $10 \%$ ) of conventional gastric adenocarcinomas (2), more than $80 \%$ of lymphoepitheliomalike carcinomas (LELCs) of the stomach $(3,4)$, and $35 \%$ of adenocarcinomas of the gastric stump are associated with Epstein-Barr virus (EBV) infection (5). The worldwide absolute number of EBV-associated gastric carcinomas makes it the largest group of EBV-associated malignancies (2). This association is characterized by the presence of EBV infection in nearly all tumor cells (6), clonality of the EBV genome in tumor cells and an elevation of EBV-specific antibodies in patients $(7,8)$. The molecular characteristics of EBVassociated gastric carcinomas have been explored. Aberrant hypermethylation of $\mathrm{CpG}$ islands is one mechanism of tumor suppressor gene inactivation in EBV-associated gastric carcinomas (9). Allelic loss at some chromosomal markers such as 5q (APC), 17p (p53) and 18q (smad 4) was inversely correlated with an EBV association. Allelic loss at the p53 
locus is not encountered in EBV-associated gastric carcinomas, but occurs in $51 \%$ of EBV-negative gastric carcinomas (10). Microsatellite instability, a hallmark of a defective DNA mismatch repair system, is not related to the EBV status in gastric carcinomas (11). By comparative genomic hybridization, loss of $4 p, 11 p$ and $18 \mathrm{q}$ was found to be more frequent in EBV-associated gastric carcinomas than in EBV-negative gastric carcinomas (12). By a tissue array analysis, EBVassociated gastric carcinomas were shown to exhibit frequent loss of expression of some tumor suppressor genes such as p16, smad 4, FHIT and KAI-1, but usually retained the expressions of APC, DCC and some DNA-repair proteins (13). The clinicopathological features of EBV-associated gastric carcinomas are found more frequently in male and younger patients, and in the proximal part of the stomach with a tubular histopathological subtype together with prominent activated $\mathrm{CD} 8^{+}$lymphocyte infiltration and a lower frequency of lymph node metastasis $(14,15)$. EBVassociated adenocarcinomas do not obviously differ in prognosis compared to EBV-negative adenocarcinomas $(16,17)$, but the prognosis of EBV-associated LELCs of the stomach is significantly better than that of conventional EBV-negative gastric adenocarcinomas $(18,19)$. All of these findings suggest that EBV-associated gastric carcinomas exhibit specific clinicopathological features, novel genomic and epigenetic aberrations, and a distinct protein expression profile. Therefore, EBV-associated gastric carcinomas have a distinct pathogenic pathway in which EBV infection and its Epstein-Barr nuclear antigen 1 (EBNA-1) might play crucial roles.

In EBV-associated gastric carcinomas, EBV shows a unique latent pattern characterized by transcription of the transforming BARF1 gene, EBER1/2, Q-promoter-driven EBNA1, BARF0 and LMP2A, but not EBNA-2, -3A, -3B, or $-3 \mathrm{C}$, leader protein, or other latent membrane proteins (LMPs) due to gene methylation $(7,20,21)$. EBNA1 is a DNA-binding nuclear phosphoprotein essentially required for replication and maintenance of the episomal EBV genome through the binding of EBNA1 to the origin of viral replication (22). EBNA1 is consistently expressed in EBV-associated gastric carcinomas and can act as a transcriptional transactivator upregulating the $\mathrm{Cp}$ and LMP1 promoters (23). EBNA1 also interacts with the Qp promoter and immediately downstream regulatory region III to positively and negatively regulate its own expression (24). The EBNA1 protein can be separated into amino- and carboxy-terminal domains by a glycineglycine-alanine repeat (GAr) sequence, which acts as a cisacting inhibitor of MHC class I-restricted presentation and appears to function by inhibiting antigen processing via the ubiquitin/proteasome pathway $(25,26)$. In addition, EBNA1 has a well-defined modular structure with no apparent enzymatic activity, but may fulfill its functions through interactions with cellular proteins. Many cellular-interacting proteins such as importin- $\alpha$, karyopherin- $\alpha 1$, P32/TAP, EBP2, TAFI $\alpha$, TAF-I 3, CK2, PRMT5 and USP7 were shown to interact with EBNA1 (27-32), but their exact biological effects are not well clarified.

Several reports supported the hypothesis that EBNA1 has oncogenic potential, and these include the fact that EBNA1 is required as a survival factor in Burkitt's lymphoma (33), that only EBNA1 is consistently expressed in EBV-associated Burkitt's lymphoma and gastric carcinomas (34), and that EBNA1 is able to induce B-cell neoplasia in transgenic mice (35). Moreover, we showed that the expression of EBNA-1 in an EBV-negative NPC cell line resulted in increased tumorigenic and metastatic capabilities in vivo (36), thus suggesting that EBNA-1 may enhance the malignant progression of EBV-associated epithelial tumors. These findings suggest that EBNA-1 is the EBV-encoded protein consistently expressed in all EBV-associated malignancies, which has biological effects and as such would seem to play a critical role in viral persistence and EBV-mediated cellular transformation (37). In contrast, it was also shown that EBNA1 is unable to induce lymphomas in transgenic FVB mice (38) and may act as a transforming suppressor of the HER2/neu oncogene by its N-terminal domain (39). Furthermore, it is able to sensitize HER2/neu-overexpressing ovarian cancer cells to topoisomerase II-targeted and paclitaxel drugs (40). In addition, the expression of EBNA1 in epithelial cells requires an undifferentiated cellular environment and may induce cytotoxic effects in some cell lines (41). All of these divergent findings suggest that the exact biological activities of EBNA1 may be complicated and result from a diverse range of biological effects on different cellular systems together with interactions with various extracellular environmental factors.

Based on the restricted EBV gene expression in gastric epithelial cells, EBNA1 is the latent protein consistently expressed in all EBV-associated gastric carcinomas. Although EBNA1 would seem to contribute at least some pathogenic factors to tumorigenesis and/or the modulation of clinicopathological features of EBV-associated gastric carcinomas, its biological effects in gastric carcinoma are still not well understood. In this report, using in vivo and in vitro studies, we evaluated the oncogenic potential and alterations in cisplatin sensitivity between two gastric carcinoma cell lines (SCM1 and TMC1) that were transfected with a gene expressing EBNA1.

\section{Materials and methods}

Cells and animals. The EBV-negative gastric carcinoma cell lines SCM1 and TMC1 used in this study are derived from gastric adenocarcinomas and were cultured in RPMI-1640 medium containing $10 \%$ fetal calf serum (FCS) as previously described (42). The LL/2 (LLC1) cell line derived from a primary Lewis lung adenocarcinoma (43) was cultured in Dulbecco's modified Eagle's medium (DMEM) with $4 \mathrm{mM}$ L-glutamine and $1 \mathrm{mM}$ sodium pyruvate containing $10 \%$ fetal bovine serum (FBS). All cell lines were incubated at $37^{\circ} \mathrm{C}$ in an incubator with $5 \% \mathrm{CO}_{2}$ and water-saturated air. Pathogen-free nude mice (Balb/c nu/nu) and immunocompetent $\mathrm{Balb} / \mathrm{c}$ mice were used for the tumorigenic test, which involved subcutaneous transplantation of either EBNA1expressing gastric carcinoma cells, EBNA1-expressing LL/2 cells, or vector-transfected cells. All procedures involving animal experiments and the care of animals in this study carried out in accordance with an established animal protocol and were approved by the Experimental Animal Care and Use Committee at National Defense Medical Center. 
Plasmid construction and DNA transfection. The EBNA1 gene was introduced into the pCP4/EBNA1 plasmid and the control pCP4 vector, and the DNA transfection methods are as previously described (44). The pCP4/EBNA1 plasmid was used to transfect SCM1, TMC1 and LL/2 cells to establish the EBNA1-expressing cells to evaluate the biological effects of EBNA1 in transfected cells. pCP4 was used to establish vector-transfected control cells. After transfection for $24 \mathrm{~h}$, transfected SCM1 and TMC1 cells were subcultured at a 1:10 dilution into selection medium consisting of growth medium containing $100 \mu \mathrm{g} / \mathrm{ml}$ hygromycin, while transfected LL/2 cells were subcultured in selection medium consisting of growth medium containing $20 \mu \mathrm{g} / \mathrm{ml}$ hygromycin. After long-term selection, stable transfected cells were established.

Immunocytochemical staining. EBNA1 expression in transfected SCM1, TMC1 and LL/2 cells was detected by in situ immunocytochemical staining, as previously described with minor modifications (42). For immunocytochemical staining, normal goat serum (at a 1:20 dilution in TBS) was used as the blocking reagent. Cells were then incubated with antiserum (at a 1:30 dilution) from an EBV-positive donor with nasopharyngeal carcinoma (NPC) for $2 \mathrm{~h}$; this was followed by incubation with a biotin-labeled goat anti-human IgG antibody (Santa Cruz, CA, USA), then streptavidin was linked to horseradish peroxidase (Vector, Burlingame, CA, USA). Slides were washed three times in TBS for 5 min each. The color was developed in AEC solution (Vector), and slides were slightly counterstained with hematoxylin and mounted for examination. Substitution of the primary antibody with neonatal umbilical cord serum or omission of the primary antibody served as a negative control for all immunostaining.

Western immunoblotting. Western immunoblotting was used to evaluate EBNA1 expression in the established EBNA1expressing SCM1, TMC1 and LL/2 cells, as previously described with minor modifications (44). In brief, blots were incubated with blocking buffer containing 2\% BSA, $1 \%$ normal goat serum and $0.1 \%$ Tween-20 in TBS for $45 \mathrm{~min}$ and then incubated with antiserum (at a 1:200 dilution) from an EBV-positive donor with NPC for $2 \mathrm{~h}$. The antigenantibody complex was detected by an HRP-coupled goat anti-human immunoglobulin G (IgG) antibody (Santa Cruz) followed by Western Blotting Luminol Reagent (Santa Cruz). The same procedures were used to evaluate the expressions of GST $\pi$, MDR1, topoisomerase I and II, Her-2/neu, and p53 in EBNA1-expressing gastric carcinoma cells by respectively substituting the primary antibodies with anti-human GST $\pi$ (Novocastra, UK), MDR1 (Santa Cruz), topoisomerase I and II (NeoMarkers, CA USA), Her-2/neu (NeoMarkers) and p53 (DO7, NeoMarkers).

Immunoprecipitation of EBNA1. Immunoprecipitation plus Western immunoblotting was used to evaluate EBNA1 expression in tumor masses derived from the tumorigenic assay in nude mice or Balb/c mice as described in a previous report with minor modifications $(36,44)$. In brief, the extracted protein concentration was determined by a bicinchonic acid assay (Pierce, Rockford, IL, USA), and an appropriate amount of protein was used for immunoprecipitation. Proteins were pre-cleared by adding neonatal umbilical cord serum (at a 1:30 dilution) and $1.0 \mu \mathrm{g}$ of normal goat IgGs together with $30 \mu \mathrm{l}$ of protein $\mathrm{A} / \mathrm{G}$-agarose $(25 \% \mathrm{v} / \mathrm{v})$; this was followed by incubation at $4^{\circ} \mathrm{C}$ for $30 \mathrm{~min}$, and then centrifugation at $13,000 \mathrm{rpm}$ for $5 \mathrm{~min}$ at $4^{\circ} \mathrm{C}$. The supernatant was transferred to a fresh tube on ice, and immunoprecipitation was initiated by the addition of antiserum (at a 1:30 dilution) from an EBV-positive donor with NPC, followed by incubation overnight at $4^{\circ} \mathrm{C}$. Incubation was then carried out for an additional $4 \mathrm{~h}$ in the presence of protein $\mathrm{A} / \mathrm{G}$ agarose. The immunoprecipitate was washed four times in lysis buffer. For sodium dodecylsulfate polyacrylamide gel electrophoresis (SDSPAGE), pellets were resuspended in $30 \mu \mathrm{l}$ of $3 \mathrm{x}$ SDS sample buffer and then heated at $98^{\circ} \mathrm{C}$ for $5 \mathrm{~min}$, followed by SDSPAGE. For the Western immunoblot analysis, proteins were transferred to nitrocellulose; membranes were blocked with $3 \%$ BSA and $0.1 \%$ Tween-20 in TBS for $90 \mathrm{~min}$ and incubated with antiserum (at 1:300 dilution) from an EBVpositive donor with NPC. The antigen-antibody complex was detected by HRP-coupled goat anti-human IgG antibody (Santa Cruz) followed by luminol reagent (Santa Cruz).

MTT assay. The MTT assay procedures used to evaluate the difference in growth rates between EBNA1-expressing cells and vector-transfected control cells were the same as those previously described (45).

Tumorigenic assay in nude and Balb/c mice. The tumorigenic assay in nude mice was used to evaluate variations in tumorigenesis between EBNA1-expressing SCM1 and TMC1 cells and vector-transfected SCM1 and TMC1 cells, respectively, as previously described (42). In brief, $4 \times 10^{6}$ cells were subcutaneously transplanted into the right and left back regions of each nude mouse. Animals were sacrificed on the 60 th day of the experiment, and the tumor mass was excised, weighed and divided into two parts. One of them was fixed in $10 \%$ neutralized formalin for the histopathological examination, and the other was quickly frozen in liquid nitrogen then stored at $-80^{\circ} \mathrm{C}$ for studies such as the detection of EBNA1 expression in tumor cells by immunoprecipitation and Western immunoblotting. The tumorigenicity of EBNA1expressing LL/2 cells and vector-transfected LL/2 cells was evaluated by an allograft in Balb/c mice according to the same experimental procedures.

Morphological examination. The morphological characteristics of cultured EBNA1-expressing cells and vectortransfected control cells were examined under subconfluent culture conditions. The morphological features of each tumor mass derived from the tumorigenicity assay were evaluated by serial tissue sections using hematoxylin and eosin $(\mathrm{H} \& \mathrm{E})$ staining.

Clonogenic assay. A clonogenic assay was used to evaluate differences in cisplatin sensitivity between EBNA1-expressing and vector-transfected gastric carcinoma cells as previously described with modifications (46). Cells growing in the log phase were trypsinized, and viable cells were counted. On a 60-mm culture dish, appropriate cell numbers (1000 SCM1 cells and $2000 \mathrm{TMC} 1$ cells) were seeded in selection medium. 

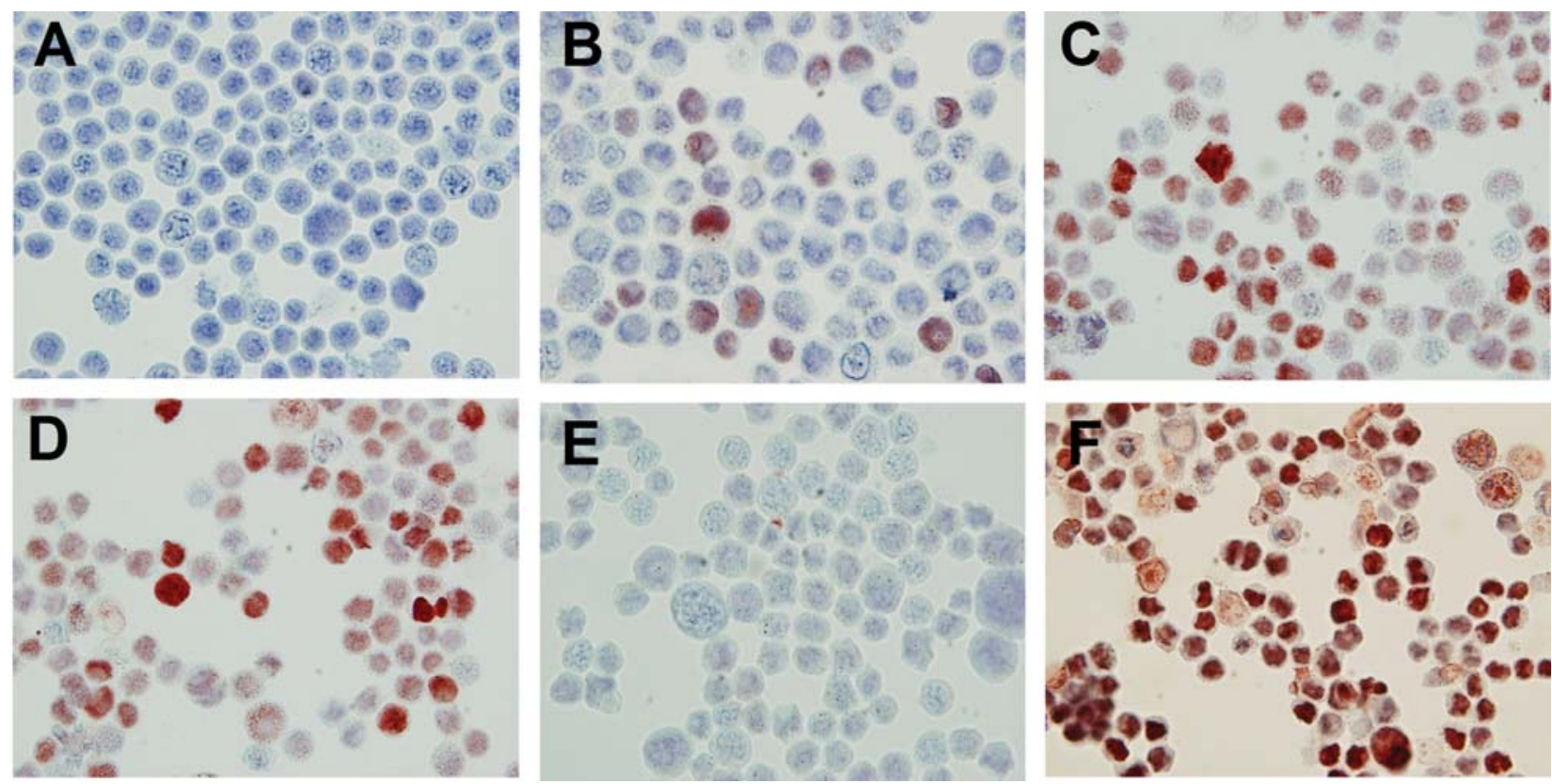

Figure 1. Immunocytochemical staining for EBNA1 expression in EBNA1-expressing gastric carcinoma cells. (A) Vector-transfected SCM1 cells. (B) EBNA1expressing SCM1 cells. (C) Subclonal EBNA1-expressing SCM1 cells (B8). (D) Subclonal EBNA1-expressing SCM1 cells (D23). (E) Vector-transfected TMC1 cells. (F) EBNA1-expressing TMC1 cells.

After a 24-h culture, cells was treated with various concentrations of cisplatin $(0,1,2,4,8$ and $16 \mu \mathrm{g} / \mathrm{ml})$ for $20 \mathrm{~min}$, and then washed with serum-free medium five times to remove the drug. Cisplatin-treated cells were cultured in selection medium, and the medium was refreshed every 2-3 days. After 2 weeks of incubation, cultured cells were washed with PBS, fixed in $10 \%$ neutral buffered formalin and stained with a $1 \%$ solution of crystal violet to determine the colony number. Each assay was carried out in triplicate and consisted of three independent experiments. Colonies that consisted of more than 20 cells were scored under an inverted microscope, and the number was compared to the untreated control. Cell survival curves were drawn by plotting the means of three independent experiments with the SD as an error bar.

Single-stranded conformation polymorphism (SSCP) analysis. The SSCP analysis was used to detect mutations of the p53 gene in SCM1 and TMC1 cells. It was performed using the same procedures as previously reported (47). In brief, each exon 5-8 of the p53 gene was amplified by 35 cycles of PCR using 5'-end-labeled primers and Taq polymerase (Perkin-Elmer/Cetus, Norwalk, CT, USA) and analyzed on $6 \%$ polyacrylamide gels after denaturation of the PCR products by heating at $95^{\circ} \mathrm{C}$ for $7 \mathrm{~min}$ in the presence of a $15 \%$ urea solution. Then, abnormal band shifts were demonstrated if the p53 gene had mutated exons.

Statistical analysis. Variations in tumorigenesis between EBNA1-expressing cells and vector-transfected cells were tested by Pearson's Chi-square test. Growth rates of tumors (assessed by tumor weight) induced by EBNA1-expressing cells and vector-transfected cells were evaluated by the Wilcoxon signed-rank test. Probability values of $<0.05$ were considered statistically significant.

\section{Results}

Establishment of EBNA1-expressing cells. After transfection and selection for 2 weeks, more than 300 drug-resistant colonies were obtained from the pCP4/EBNA1-transfected SCM1 and TMC1 cells, and only about 80 colonies were obtained from pCP4-transfected SCM1 and TMC1 cells. These colonies were harvested and expanded in selection medium to give EBNA1-expressing SCM1 and TMC1 cells and vector-transfected SCM1 and TMC1 cell lines. The expression of EBNA1 in EBNA1-expressing SCM1 and TMC1 cells was evaluated by in situ immunocytochemical staining and Western blotting. About 30 and 90\% of EBNA1expressing SCM1 and TMC-1 cells, respectively, showed detectable EBNA1 expression in the nucleus by in situ immunocytochemical staining (Fig. 1). By Western blotting, EBNA1-expressing SCM1 and TMC1 cells expressed EBNA1 at $83 \mathrm{kDa}$ (Fig. 2A). Because the expression level of EBNA1 in tumor cells was too low for detection by in situ immunocytochemical staining or the Western blotting method, EBNA1 expression in the tumors derived from the tumorigenic assay of EBNA1-expressing SCM1 and TMC1 cells was evaluated by immunoprecipitation plus Western blotting (Fig. 2B). Vector-transfected SCM1 and TMC1 cells showed no EBNA1 expression in either system. In addition, EBNA1expressing LL/2 cells and vector-transfected LL/2 cells were established by the same procedure. EBNA1 expression in EBNA1-expressing LL/2 cells was undetectable by in situ immunocytochemical staining due to its lower-level expression. However, EBNA1 expression could also be detected as an $83-\mathrm{kDa}$ protein in EBNA1-expressing LL/2 cells by Western blotting and in the tumor mass derived from the tumorigenic assay by immunoprecipitation plus Western blotting (Fig. 2A and B). These results confirmed that SCM1, 
Table I. Tumorigenesis of EBNA1-expressing gastric carcinoma cells and vector-transfected gastric carcinoma cells in nude mice, and tumorigenesis of EBNA1-expressing LL/2 cells and vector-transfected LL/2 cells in Balb/c mice.

\begin{tabular}{|c|c|c|c|c|c|c|}
\hline $\begin{array}{l}\text { Mouse } \\
\text { number }\end{array}$ & $\begin{array}{l}\text { EBNA1- } \\
\text { expressing SCM1 } \\
\text { cell-derived } \\
\text { tumors }(\mathrm{g})\end{array}$ & $\begin{array}{c}\text { Vector- } \\
\text { transfected SCM1 } \\
\text { cell-derived } \\
\text { tumors }(\mathrm{g})\end{array}$ & $\begin{array}{l}\text { EBNA1- } \\
\text { expressing TMC1 } \\
\text { cell-derived } \\
\text { tumors }(\mathrm{g})\end{array}$ & $\begin{array}{c}\text { Vector- } \\
\text { transfected TMC1 } \\
\text { cell-derived } \\
\text { tumors }(\mathrm{g})\end{array}$ & $\begin{array}{l}\text { EBNA1- } \\
\text { expressing LL/2 } \\
\text { cell-derived } \\
\text { tumors }(\mathrm{g})\end{array}$ & $\begin{array}{l}\text { Vector- } \\
\text { transfected LL/2 } \\
\text { cell-derived } \\
\text { tumors }(\mathrm{g})\end{array}$ \\
\hline 1 & 1.03 & 0.3 & 2.39 & 0.38 & 5.17 & 0 \\
\hline 2 & 0.14 & 0 & 0.89 & 0.03 & 0 & 0 \\
\hline 3 & 0.16 & 0.40 & 0.76 & 0 & 0 & 0 \\
\hline 4 & 2.31 & 0.45 & 1.05 & 0 & 0 & 0 \\
\hline 5 & 0.27 & 0 & 0.3 & 0 & 0 & 0 \\
\hline 6 & 0.59 & 0.54 & 0.55 & 0.46 & 1.85 & 0 \\
\hline 7 & 1.09 & 0.82 & 1.09 & 0 & 3.22 & 0 \\
\hline 8 & 2.68 & 1.10 & 0 & 0 & 0 & 0 \\
\hline 9 & 3.42 & 2.01 & 0 & 0 & 0 & 0 \\
\hline 10 & 1.52 & 0.74 & 0 & 0 & 0 & 0 \\
\hline 11 & 0.59 & 0 & 0.59 & 0 & 2.65 & 0 \\
\hline 12 & 1.25 & 0.83 & 1.25 & 0.83 & 0 & 0 \\
\hline $\begin{array}{l}\text { Mean of tumor } \\
\text { weight }(g)^{\mathrm{a}}\end{array}$ & 1.25 & 0.74 & 0.98 & 0.43 & 3.78 & 0 \\
\hline Tumorigenicity $(\%)^{\mathrm{b}}$ & 100 & 75 & 75 & 33 & 33 & 0 \\
\hline
\end{tabular}

${ }^{a}$ Wilcoxon signed-rank test for the growth rate (assessed by tumor weight); the difference was significant at $\mathrm{p}<0.05$. ${ }^{\mathrm{b}} \mathrm{Pearson}$ Chi-square test for tumorigenicity; the difference was significant at $\mathrm{p}<0.05$.

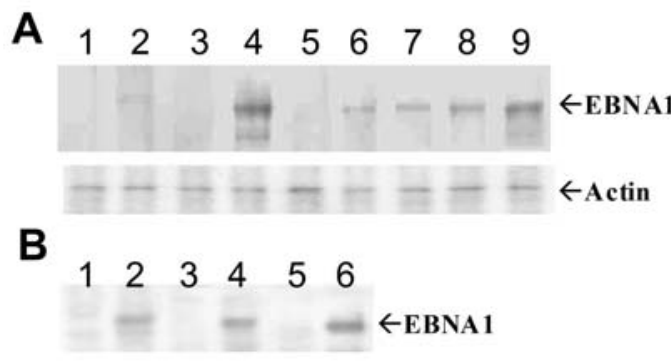

Figure 2. Western blot analysis of EBNA1 expression in EBNA1-expressing LL/2, TMC1 and SCM1 cells. (A) EBNA1 expression in EBNA1expressing cells demonstrated by Western blotting; aliquots of $20 \mu \mathrm{g}$ of cell extract from each representative cell line were used. Lane 1, vectortransfected LL/2 cells; lane 2, EBNA1-expressing LL/2 cells; lane 3, vectortransfected TMC1 cells; lane 4, EBNA1-expressing TMC1 cells; lane 5, vector-transfected SCM1 cells; lane 6, EBNA1-expressing SCM1 cells; lane 7, subclonal EBNA1-expressing SCM1 cells (B15); lane 8, subclonal EBNA1expressing SCM1 cells (D8); lane 9, subclonal EBNA1-expressing SCM1 cells (D23). (B) EBNA1 expression in tumors derived from EBNA1expressing cells in a tumorigenic assay demonstrated by immunoprecipitation plus Western blotting; aliquots of $20 \mathrm{mg}$ of tumor tissue extract from each representative tumor were used except in lane 1. Lane 1, wholecell lysate of vector-transfected LL/2 cells; lane 2, tumor derived from EBNA1-expressing LL/2 cells; lane 3, tumor derived from vectortransfected SCM1 cells; lane 4, tumor derived from EBNA1-expressing SCM1 cells; lane 5, tumor derived from vector-transfected TMC1 cells; lane 6, tumor derived from EBNA1-expressiong TMC1 cells.

TMC1 and LL/2 cells transfected with EBNA1 gene are able to express EBNA1 in vitro and in vivo, but no such expression occurred in vector-transfected SCM1, TMC1 or LL/2 cells.
Growth rate of EBNA1-expressing cells. In the MTT assay, EBNA1-expressing SCM1 and TMC1 cells displayed no significant difference in growth rates when, respectively, compared to vector-transfected SCM1 and TMC1 cells (data not shown). These results suggest that EBNA1 does not obviously influence the growth ability of gastric carcinoma cells in vitro. A similar result was also obtained for EBNAexpressing LL/2 cells and vector-transfected LL/2 cells by the same assay.

Increased tumorigenicity and growth rate of EBNA1expressing cells. Results of the tumorigenic assay are summarized in Table I. Although the difference did not reach statistical significance $(\mathrm{p}=0.06)$, tumorigenesis of EBNA1expressing SCM1 cells (100\%) showed a higher frequency than that of vector-transfected SCM1 cells (75\%). The tumorigenesis frequency of EBNA1-expressing TMC1 cells $(75 \%)$ was significantly higher than that of vector-transfected TMC1 cells $(33 \%)(\mathrm{p}=0.04)$. Growth rates of tumors derived from EBNA1-expressing SCM1 and TMC1 cells, as assessed by the tumor weight, were respectively significantly higher than those of tumors derived from vector-transfected SCM1 $(\mathrm{p}=0.005)$ and TMC1 $(\mathrm{p}=0.008)$ cells. These results suggest that EBNA1-expressing gastric carcinoma cells are able to enhance tumorigenicity and growth rates compared to vectortransfected gastric carcinoma cells in an immunocompromised nude mice model. In the clinic, patients with EBVassociated gastric carcinoma always have a normal immune function (7). To clarify whether EBNA1 causes EBNA1expressing cells to have increased tumorigenicity in an immu- 
nocompetent host, we evaluated the tumorigenicity and growth rates of EBNA1-expressing LL/2 cells using allografts in immunocompetent Balb/c mice. We found that EBNA1expressing LL/2 cells were able to induce tumors (33\%), and these had the ability to grow aggressively. In contrast, no tumorigenesis was observed in vector-transfected LL/ 2 cells $(\mathrm{p}=0.01)$. These results suggest that EBNA1-expressing LL/2 cells have increased tumorigenicity and growth ability compared to vector-transfected LL/ 2 cells. Therefore, we concluded that the expression of EBNA1 is able to help host cells escape from allograft rejection and enhance tumorigenicity in an immunocompetent host.

Morphological alterations in EBNA1-expressing TMC1 cells in vivo. Morphological alterations of EBNA1-expressing gastric carcinomas and LL/2 cells were examined in vitro and in vivo. Under subconfluent culture conditions, there was no obvious difference in morphological alterations between EBNA1-expressing SCM1, TMC1 and LL/2 cells and the respective vector-transfected SCM1, TMC1 and LL/2 cells (data not shown). Morphological alterations of induced tumors in the tumorigenic tests were also evaluated using serial sections with H\&E staining. The morphological features of tumors derived from EBNA1-expressing SCM1 cells were similar to those of tumors derived from vector-transfected SCM1 cells (data not shown), whereas tumors derived from EBNA1-expressing TMC1 cells displayed a higher histological grade than tumors derived from the vector-transfected TMC1 cells, and this was characterized by greater cellular polymorphism with occasional anaplastic large cell formation, conspicuous nucleoli and frequent mitosis (Fig. 3). These findings suggest that the expression of EBNA1 in gastric carcinoma cells has the potential to induce tumors of a higher histological grade compared to tumors derived from vectortransfected gastric carcinoma cells (as observed in EBNA1expressing TMC1 cells) in the nude mouse model used.

Reduced sensitivity to cisplatin of EBNA1-expressing SCMI cells. We also evaluated the potential of EBNA1 to modulate the chemodrug sensitivity of gastric carcinoma cells by a clonogenic assay, because patients with gastric carcinoma usually receive cisplatin as adjuvant therapy. For this assessment, three additional subclonal EBNA1-expressing SCM1 cell lines (B15, D8 and D23) were isolated from EBNA1expressing SCM1 cells. In each subclonal cell line, about $80 \%$ of tumor cells expressed EBNA1 as an $83-\mathrm{kD}$ protein in the nuclei as demonstrated by in situ immunocytochemical stain and Western immunoblotting (Figs. 1 and 2A). In the clonogenic assay, each subclonal EBNA1-expressing SCM cell line (B15, D8 and D23) and EBNA1-expressing SCM1 cells displayed reduced sensitivity to cisplatin compared to vector-transfected SCM1 cells as measured by their $\mathrm{IC}_{50}$ values (a concentration that inhibited $50 \%$ of the colonyforming ability) (Fig. 4A and C). EBNA1-expressing TMC1 cells did not show an obvious change in sensitivity to cisplatin compared to vector-transfected TMC1 cells (Fig. 4B and $C$ ). These findings suggest that the expression of EBNA1 in gastric carcinoma cells has the potential to down-modulate sensitivity to cisplatin (as observed in EBNA1-expressing SCM1 cell lines).
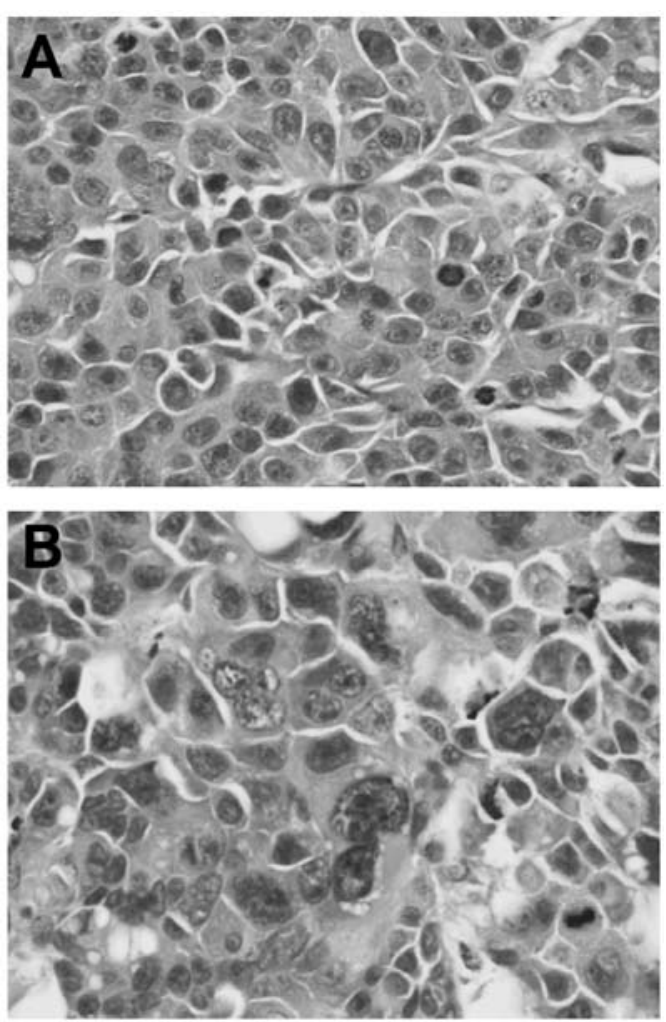

Figure 3. Histopathology of induced tumors derived from vector-transfected TMC1 cells showing a homogeneous appearance of tumor cells (A) and from the EBNA1-expressing TMC1 cells showing a higher grade of pleomorphic and anaplastic features of tumor cells (B).

Expression of drug resistance-related proteins in EBNA1expressing gastric carcinoma cells. To clarify the mechanism responsible for the reduced cisplatin sensitivity in EBNA1expressing gastric carcinoma cells, expressions of proteins related to chemodrug resistance such as GST $\pi$, MDR1 (Pglycoprotein), topoisomerase I and II, Her-2/neu and p53 were evaluated by Western blotting (48). No obvious changes in the expressions of these drug resistance-related proteins were respectively demonstrated between EBNA1-expressing SCM1 and TMC1 cells and vector-transfected SCM1 and TMC1 cells except for the p53 protein. We found that the expression level of p53 was much lower in SCM1 cells than that in TMC1 cells (Fig. 5). Immunohistochemical staining demonstrated p53 as a diffuse strong nuclear positive signal in induced tumors derived from TMC1 cells in nude mice, but in induced tumors derived from SCM1 cells, the results were negative for p53 staining (data not shown). An SSCP analysis was used to evaluate p53 mutations in SCM1 and TMC1 cells, and results showed an abnormal band shift in exon 5 of the p53 gene in TMC1 cells, but none was found in SCM1 cells (Fig. 6). These findings indicate that the p53 gene in TMC1 cells is a mutant, but it is a wild-type in SCM1 cells.

Reduced p53 expression in EBNA1-expressing SCM1 cells. EBNA1 was demonstrated to compete with p53 for binding with ubiquitin-specific protease 7 (USP7). This binding allows p53 ubiquitination and degradation by the proteosome system. Such competition is able to protect cells from apoptotic challenge and provide a mechanism to explain how 

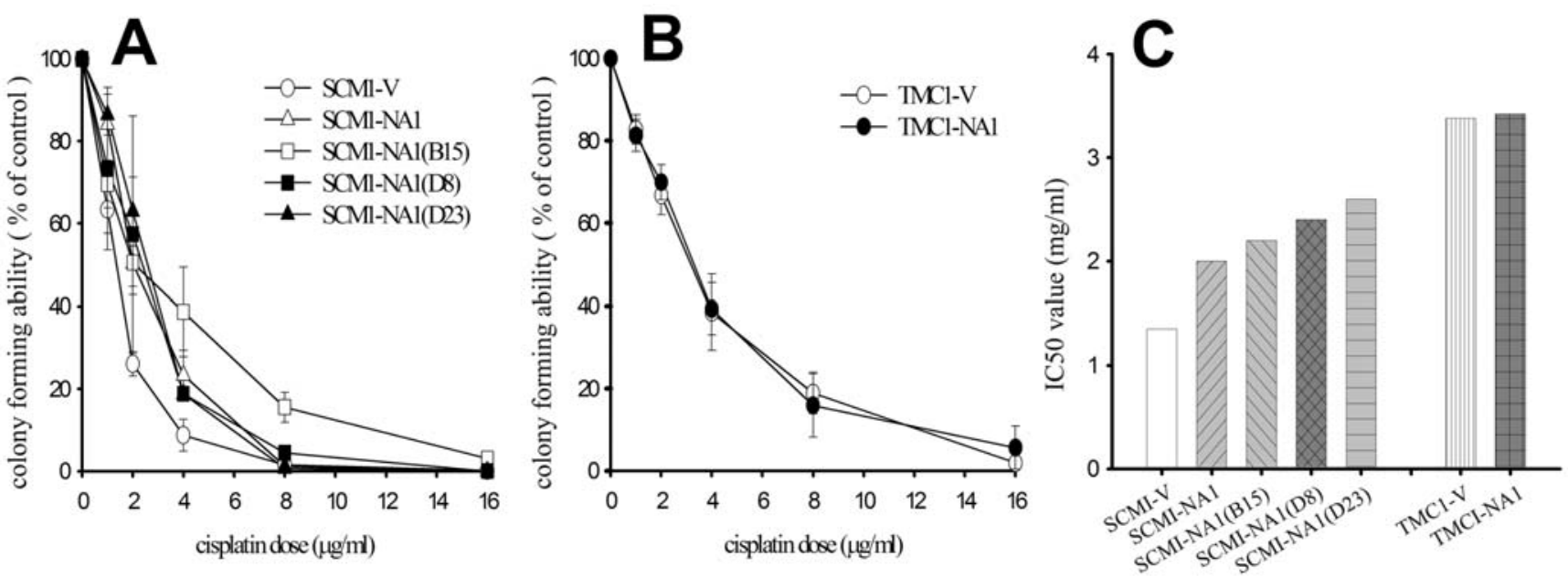

Figure 4. Clonogenic assay demonstrating the difference to cisplatin sensitivity $\left(\mathrm{IC}_{50}\right.$ value) between vector-transfected gastric carcinoma cells and EBNA1expressing gastric carcinoma cells. (A) SCM1-V, vector transfected SCM1 cells; SCM1-NA1, EBNA1-expressing SCM1 cells; SCM1-NA1(B15), SCM1NA1(D8) and SCM1-NA1(D23), different subclones of EBNA1-expressing SCM1 cells. (B) TMC1-V, vector-transfected TMC1 cells; TMC1-NA1, EBNA1expressing TMC1 cells. (C) The IC50 value of cisplatin for the EBNA1-expressing SCM1 cells and each subclonal EBNA1-expressing SCM1 cells and vector transfected SCM1 cells, and the EBNA1-expressing TMC1 cells and vector-transfected TMC1 cells are presented in the bar chart.

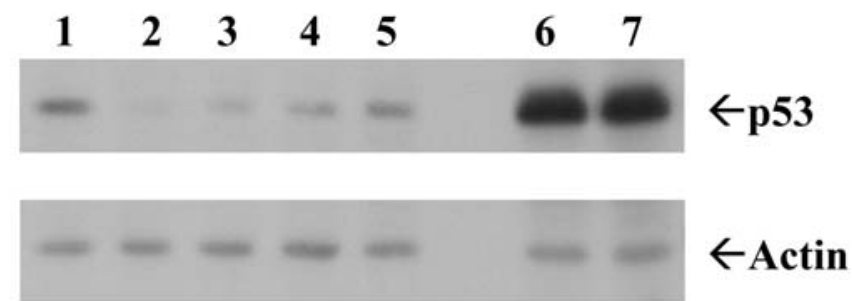

Figure 5. p53 expression in the EBNA1-expressing SCM1 cells and each subclonal EBNA1-expressing SCM1 cells and vector-transfected SCM1 cells, and the EBNA1-expressing TMC1 cells and vector-transfected TMC1 cells were demonstrated by Western blotting. Lane 1, vector-transfected SCM1 cells; lane 2, subclonal EBNA1-expressing SCM1cells (D23); lane 3, subclonal EBNA1-expressing SCM1 cells (D8); lane 4, subclonal EBNA1expressing SCM1 cells (D15); lane 5, EBNA1-expressing SCM1 cells; lane 6, vector-transfected TMC1cells; lane 7, EBNA1-expressing TMC1 cells.

EBNA1 might contribute to the survival and transformation potential of EBV-infected cells (49-52). We demonstrated that EBNA1-expressing SCM cells have reduced sensitivity to cisplatin compared to vector-transfected SCM1 cells, which possibly resulted from interactions between EBNA1 and p53. To elucidate this possibility, we evaluated the difference in p53 expression levels between EBNA1-expressing gastric carcinoma cells and vector-transfected gastric carcinoma cells. In Fig. 5, we found that the expression level of p53 was reduced in EBNA1-expressing SCM1 cells and in each subclonal EBNA1-expressing SCM1 cell line (D23, D8 and D15). In addition, the reduced p53 level was correlated with the expression level of EBNA1 in EBNA1-expressing SCM1 cells and each subclonal EBNA1-expressing SCM1 cell line (D23, D8 and D15) as shown in Fig. 2A. These findings are compatible with EBNA1 being able to compete with p53 for binding with USP7 and enhance p53 ubiquitination and degradation by the proteosome system.

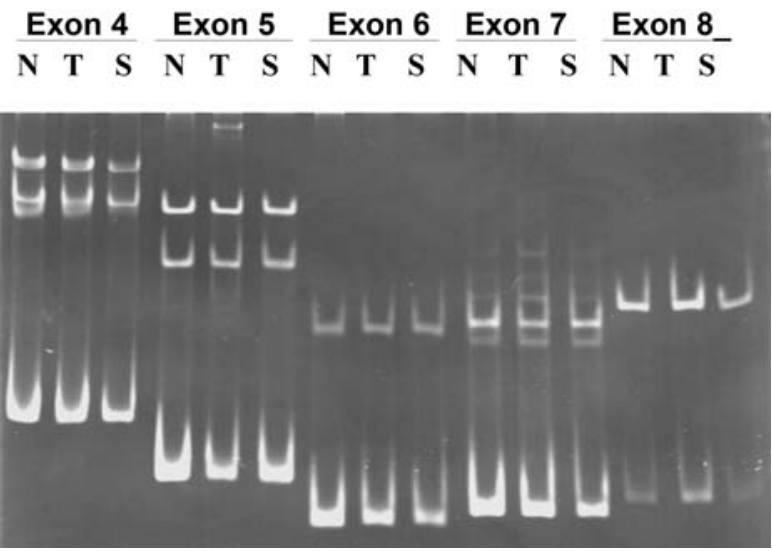

Figure 6. Single-stranded conformation polymorphism (SSCP) analysis of the $p 53$ mutation in exons $4,5,6,7$ and 8 of SCM1 and TMC1 cells. p53 gene mutation in exon 5 was observed in TMC1 cells. $\mathrm{N}$, normal control; $\mathrm{T}$, TMC1 cells; S, SCM1 cells.

\section{Discussion}

In the present study, we assessed the biological effects of EBNA1 in gastric carcinoma cells in vitro and in vivo. We found that the expression of EBNA1 in SCM1 and TMC1 cells did not obviously influence their growth ability in vitro. Similar studies demonstrated that the expression of EBNA1 is able to induce cytotoxicity in squamous epithelial cells but not in glandular epithelial cells (41). The cytotoxicity of EBNA1 was also shown in SKOV3 ovarian cancer cells which exhibit growth inhibition and G2/M arrest $(39,40)$. Based on previous reports and our results, we suggest that the expression of EBNA1 in epithelial cells seems to be able to induce diverse cytotoxicity possibly resulting from various states of the cellular environment.

With xenografts in nude mice, the expression of EBNA1 in SCM1 and TMC1 gastric carcinoma cells was able to 
enhance the tumorigenicity and growth rate, but, however, induced a higher histopathological grade of the tumor only in TMC1 cells. These results suggest that EBNA1 expression has the potential to provide advantages that promote tumorigenesis and the growth ability of EBV-associated gastric carcinomas in vivo $(22,33)$. Using allografts into immunocompetent Balb/c mice, we also showed that the expression of EBNA1 in LL/2 cells was able to induce tumors (33\%) with aggressive growth ability, but that there was no observable tumorigenesis in vector-transfected LL/2 cells. These results further suggest that EBNA1 also helps EBNA1-expressing cells escape from allograft rejection in an immunocompetent host, and this is possibly mediated by down-modulation of allograft rejection responses (53), avoidance of tumor immune surveillance mediated by the suppression of HLA molecules/ TAA on the tumor cell surface, the production of immunosuppressive cytokines, or the expression of lymphotoxic molecules (i.e., FAS ligand) by cancer cells (54-56), but these remain to be clarified in future studies. The experimental tumorigenicity results provide evidence that supports the hypothesis that expression of EBNA1 in EBV-associated gastric carcinoma may play an important role in tumorigenesis and a tumor's ability to escape from host immune eradication during EBV-mediated cell transformation. In previous reports, the oncogenic potential of EBNA1 was also identified in other cellular systems including the induction of lymphomagenesis in transgenic mice $(35,57)$ and the enhancement of malignant progression of NPC cells in vivo (36) at low-level expression only detected by immunoprecipitated Western blotting. However, EBNA1 failed to induce lymphomas in transgenic FVB mice at an expression level similar to that of latent EBV infection in human B lymphocytes (38). These discrepant findings might have resulted from different expression levels of EBNA1. The GAr of EBNA1 can protect EBNA1 from proteasomal degradation and results in a failure of presentation of EBNA1derived epitopes on MHC class I molecules and therefore in $\mathrm{CD}^{+} \mathrm{T}$ cell recognition. This helps latently infected cells escape from cell-mediated immune responses during the transformation or long-term survival in vivo $(25,26,58)$. Importantly, GAr also inhibits mRNA translation of EBNA1 in cis and minimizes the expression of EBNA1, both in vitro and in vivo, thus avoiding efficient presentation of respective MHC class I and II molecules for $\mathrm{CD}^{+}$and $\mathrm{CD}^{+}{ }^{+} \mathrm{T}$ cell recognition (59-61). The protective effects of GAr regulation of EBNA1 expression to a minimized stable level may allow the persistence of the virus in latently infected cells and make it more suitable to mediate cell transformation (62).

The oncogenic mechanisms of EBNA1 are not well clarified, but there are some reports of biological activities of EBNA1 that provide some reasonable explanations. EBNA1 was shown to have oncogenic potential through different action mechanisms: a) it interacts with $\mathrm{Nm} 23-\mathrm{H} 1$ and inhibits its ability to suppress cell migration in lymphoblastoid cells, which highlights a novel function of EBNA1 that contributes to malignant transformation and tumor progression (63); b) it modulates the AP-1 transcription factor pathway in nasopharyngeal carcinoma cells and enhances angiogenesis which implicates EBNA1 in the angiogenic process and might contribute to the development and aggressively metastatic nature of NPC (64); c) it promotes genomic instability via induction of reactive oxygen species, highlighting a novel function of EBNA-1 which can contribute to malignant transformation and tumor progression (65); and d) it modulates the STAT1 and TGF- $\beta$ signaling pathways to induce a lower steady-state level of SMAD2 protein, downregulates the tumor growth factor (TGF)- $\beta$ target gene, PTPRK, and contributes to the growth and survival of Hodgkin lymphoma cells, suggesting that EBNA1 can influence cellular gene transcription resulting in effects that may contribute to the development of EBV-associated tumors $(49,50)$. These observations support the view that the expression of EBNA1 in $\mathrm{EBV}$-associated tumors might not only reflect a requirement for viral persistence but also results in a selective growth advantage and increased oncogenic potential that enhances the malignant transformation and progression of tumor cells.

By a clonogenic assay, EBNA1-expressing SCM cells were shown to have reduced sensitivity to cisplatin compared to vector-transfected SCM1 cells, but the same results were not observed in EBNA1-expressing TMC1 cells. The underlying molecular mechanisms of these findings are not easily explained based on the present study, because no upregulated expression of the drug resistance-related proteins GST $\pi$, MDR1 (P-glycoprotein), topoisomerase I and II, or Her-2/neu was demonstrated in EBNA1-expressing SCM1 or TMC1 cells. However, we found that the p53 expression level was much lower in SCM1 cells than in TMC1 cells as demonstrated by the Western blot assay. By the SSCP analysis, we showed that a point mutation in the p53 gene had occurred in TMC1 cells, but the p53 gene remained a wild-type in SCM1 cells.

EBNA1-expressing SCM cells had reduced sensitivity to cisplatin compared to vector-transfected SCM1 cells, but that was not found for EBNA1-expressing TMC1 cells which is compatible with EBNA1 being demonstrated to play a role as a survival factor in Burkitt's lymphoma by being able to inhibit p53-induced apoptosis in vitro (34). These findings can be explained by EBNA1 possibly competing with p53 for binding to USP7. This binding allows p53 ubiquitination and degradation. Such competition may therefore protect cells from apoptotic challenge and provide a mechanism to explain how EBNA1 might contribute to the survival and transformation potential of EBV-infected cells $(66,67)$. Such protection from apoptotic challenge is predicated to protect cells from DNA damage induced by apoptosis mediated through p53 activity. Thus, we suggest that the expression of EBNA1 in SCM cells in parallel with wild-type p53 ubiquitination and degradation by the ubiquitin/proteasome system reduces DNA damage by apoptosis induced by cisplatin treatment. But this was not observed in TMC1 cells because the mutant p53 in TMC1 cells may be insensitive to EBNA1's ubiquitination modulation and/or the mutant p53 has greater potential than EBNA1 to reduce DNA damage induced by apoptosis. This rationally explains the expression of EBNA1 in SCM1 cells (with wild-type p53) conferring reduced sensitivity to cisplatin and why no similar finding was observed in EBNA1-expressing TMC1 cells (with mutant p53). In addition, this explanation is also compatible with clinical findings that in EBV-positive gastric carcinomas, 
infrequent overexpression of p53 protein (68) and much lower frequency of p53 gene mutation $(10,69)$ are displayed, which are significantly distinct from EBV-negative gastric carcinomas.

Finally, the present study shows that the expression of EBNA in gastric carcinoma cells has the potential to enhance tumorigenicity and induce tumors with higher-grade histopathological features (observed in TMC1 cells) in a xenograft nude mouse model. EBNA1-expressing LL/2 cells also showed enhanced tumorigenicity and growth ability in the allograft immunocompetent Balb/c mouse model. These results support the hypothesis that the expression of EBNA1 in EBV-associated gastric carcinoma may play an important role in EBV-mediated cell transformation and allow such cells to escape from a patient's immune eradication, possibly by down-modulation of tumor immune surveillance and/or rejection responses. In addition, EBNA1 is able to reduce the sensitivity to the DNA damage cytotoxicity induced by cisplatin in gastric carcinoma cells harboring wild-type p53 and suggests that EBV infection with EBNA1 expression reduces the sensitivity to apoptotic stimuli of host cells. Together, our results provide an additional example highlighting that EBV infection with EBNA1 expression provides host cells with advantages in terms of survival and transformation potential, escape from host immunosurveillance, and reduced sensitivity to the DNA-damage stress and/or apoptotic stimuli from the extracellular environment. These findings may characterize the pathogenesis of EBVpositive gastric carcinomas, which is distinct from that of EBV-negative gastric carcinomas.

\section{Acknowledgments}

This investigation was supported by grants from the National Science Council (NSC93-2320-B-016-005 and NSC94-2320B-016-001), Taipei, Taiwan. The authors sincerely thank Hsien-Chuan Chin for the excellent statistical analysis.

\section{References}

1. Parkin DM: Global cancer statistics in year 2000. Lancet Oncol 2: 533-543, 2001.

2. Takada K: Epstein-Barr virus and gastric carcinoma. J Clin Pathol Mol Pathol 53: 255-261, 2000.

3. Burke AP, Yen TS, Shekitka KM and Sobin LH: Lymphoepithelial carcinoma of the stomach with Epstein-Barr virus demonstrated by polymerase chain reaction. Mod Pathol 3: 377 380, 1990.

4. Shibata D, Tokunaga M, Uemura Y, Sato E, Tanaka S and Weiss LM: Association of Epstein-Barr virus with undifferentiated gastric carcinomas with intense lymphoid infiltration. Lymphoepithelioma-like carcinoma. Am J Pathol 139: 469-474, 1991.

5. Yamamoto N, Tokunaga M, Uemura Y, Tanaka S, Shirahama H, Nakamura T, Land CE and Sato E: Epstein-Barr virus and gastric remnant cancer. Cancer 74: 805-809, 1994.

6. Shibata D and Weiss LM: Epstein-Barr virus-associated gastric adenocarcinoma. Am J Pathol 140: 769-774, 1992.

7. Imai S, Koizumi S, Sugiura M, Tokunaga M, Uemura Y, Yamamoto N, Tanaka S, Sato E and Osato T: Gastric carcinoma: monoclonal epithelial malignant cells expressing Epstein-Barr virus latent infection protein. Proc Natl Acad Sci USA 91: 9131-9135, 1994.

8. Levine PH, Stemmermann G, Lennette ET, Hildesheim A, Shibata D and Nomura A: Elevated antibody titers to EpsteinBarr virus prior to the diagnosis of Epstein-Barr-virus-associated gastric adenocarcinoma. Int J Cancer 60: 642-644, 1995.
9. Kang GH, Lee S, Kim WH, Lee HW, Kim JC, Rhyu MG and Ro JY: Epstein-Barr virus-positive gastric carcinoma demonstrates frequent aberrant methylation of multiple genes and constitutes $\mathrm{CpG}$ island methylator phenotype-positive gastric carcinoma. Am J Pathol 160: 787-794, 2002.

10. van Rees BP, Caspers E, zur Hausen A, van den Brule A, Drillenburg P, Weterman MA and Offerhaus GJ: Different pattern of allelic loss in Epstein-Barr virus-positive gastric cancer with emphasis on the p53 tumor suppressor pathway. Am J Pathol 161: 1207-1213, 2002.

11. Chang MS, Lee HS, Kim HS, Kim SH, Choi SI, Lee BL, Kim CW, Kim YI, Yang M and Kim WH: Epstein-Barr virus and microsatellite instability in gastric carcinogenesis. J Pathol 199: 447-452, 2003.

12. zur Hausen A, van Grieken NC, Meijer GA, Hermsen MA, Bloemena E, Meuwissen SG, Baak JP, Meijer CJ, Kuipers EJ and van den Brule AJ: Distinct chromosomal aberrations in Epstein-Barr virus-carrying gastric carcinomas tested by comparative genomic hybridization. Gastroenterology 121 : 612-618, 2001.

13. Lee HS, Chang MS, Yang HK, Lee BL and Kim WH: EpsteinBarr virus-positive gastric carcinoma has a distinct protein expression profile in comparison with Epstein-Barr virusnegative carcinoma. Clin Cancer Res 10: 1698-1705, 2004.

14. van Beek J, zur Hausen A, Klein Kranenbarg E, van de Velde CJ, Middeldorp JM, van den Brule AJ, Meijer CJ and Bloemena E: EBV-positive gastric adenocarcinomas: a distinct clinicopathologic entity with a low frequency of lymph node involvement. J Clin Oncol 22: 664-670, 2004.

15. Saiki Y, Ohtani H, Naito $Y$, Miyazawa $M$ and Nagura $H$ : Immunophenotypic characterization of Epstein-Barr virusassociated gastric carcinoma: massive infiltration by proliferating CD8 ${ }^{+}$T-lymphocytes. Lab Invest 75: 67-76, 1996.

16. Gulley ML, Pulitzer DR, Eagan PA and Schneider BG: EpsteinBarr virus infection is an early event in gastric carcinogenesis and is independent of bcl-2 expression and p53 accumulation. Hum Pathol 27: 20-27, 1996.

17. Chang MS, Lee HS, Kim CW, Kim YI and Kim WH: Clinicopathologic characteristics of Epstein-Barr virus-incorporated gastric cancers in Korea. Pathol Res Pract 197: 395-400, 2001.

18. Watanabe H, Enjoji M and Imai T: Gastric carcinoma with lymphoid stroma: Its morphologic characteristics and prognostic correlations. Cancer 38: 232-243, 1976.

19. Nakamura S, Ueki T, Yao T, Ueyama T and Tsuneyoshi M: Epstein-Barr virus in gastric carcinoma with lymphoid stroma: Special reference to its detection by the polymerase chain reaction and in situ hybridization in 99 tumors, including a morphologic analysis. Cancer 73: 2239-2249, 1994.

20. Sugiura M, Imai S, Tokunaga M, Koizumi S, Uchizawa M, Okamoto $\mathrm{K}$ and Osato T: Transcriptional analysis of EpsteinBarr virus gene expression in EBV-positive gastric carcinoma: unique viral latency in the tumour cells. Br J Cancer 74: 625-631, 1996.

21. zur Hausen A, Brink AA, Craanen ME, Middeldorp JM, Meijer CJ and van den Brule AJ: Unique transcription pattern of EpsteinBarr virus (EBV) in EBV-carrying gastric adenocarcinomas: expression of the transforming BARF1 gene. Cancer Res 60: 2745-2748, 2000

22. Leight ER and Sugden B: EBNA-1: a protein pivotal to latent infection by Epstein-Barr virus. Rev Med Virol 10: 83-100, 2000.

23. Schlager S, Speck SH and Woisetschlager M: Transcription of the Epstein-Barr virus nuclear antigen 1 (EBNA1) gene occurs before induction of the BCR2 (Cp) EBNA gene promoter during the initial stages of infection in B cells. J Virol 70: 3561-3570, 1996.

24. Nonkwelo C, Skinner J, Bell A, Rickinson A and Sample J: Transcription start sites downstream of the Epstein-Barr virus (EBV) Fp promoter in early-passage Burkitt lymphoma cells define a fourth promoter for expression of the EBV EBNA-1 protein. J Virol 70: 623-627, 1996.

25. Levitskaya J, Coram M, Levitsky V, Imreh S, SteigerwaldMullen PM, Klein G, Kurilla MG and Masucci MG: Inhibition of antigen processing by the internal repeat region of the Epstein-Barr virus nuclear antigen-1. Nature 375: 685-688, 1995.

26. Levitskaya J, Sharipo A, Leonchiks A, Ciechanover A and Masucci MG: Inhibition of ubiquitin/proteasome-dependent protein degradation by the Gly-Ala repeat domain of the Epstein-Barr virus nuclear antigen 1. Proc Natl Acad Sci USA 94: 12616-12621, 1997. 
27. Kim AL, Maher M, Hayman JB, Ozer J, Zerby D, Yates JL and Lieberman PM: An imperfect correlation between DNA replication activity of Epstein-Barr virus nuclear antigen 1 (EBNA1) and binding to the nuclear import receptor, Rch1/importin alpha. Virology 239: 340-351, 1997.

28. Wang Y, Finan JE, Middeldorp JM and Hayward SD: P32/TAP, a cellular protein that interacts with EBNA-1 of Epstein-Barr virus. Virology 236: 18-29, 1997.

29. Fischer N, Kremmer E, Lautscham G, Mueller-Lantzsch N and Grasser FA: Epstein-Barr virus nuclear antigen 1 forms a complex with the nuclear transporter karyopherin alpha 2. J Biol Chem 272: 3999-4005, 1997.

30. Shire K, Ceccarelli DF, Avolio-Hunter TM and Frappier L: EBP2, a human protein that interacts with sequences of the Epstein-Barr virus nuclear antigen 1 important for plasmid maintenance. J Virol 73: 2587-2595, 1999.

31. Ito S, Ikeda M, Kato N, Matsumoto A, Ishikawa Y, Kumakubo S and Yanagi K: Epstein-Barr virus nuclear antigen-1 binds to nuclear transporter karyopherin alpha1/NPI-1 in addition to karyopherin alpha2/Rch1. Virology 266: 110-119, 2000.

32. Aiyar A, Tyree C and Sugden B: The plasmid replicon of EBV consists of multiple cis-acting elements that facilitate DNA synthesis by the cell and a viral maintenance element. EMBO J 17: 6394-6403, 1998.

33. Kennedy G, Komano J and Sugden B: Epstein-Barr virus provides a survival factor to Burkitt's lymphomas. Proc Natl Acad Sci USA 100: 14269-14274, 2003.

34. Rickinson $A B$ and Kieff E: Epstein-Barr virus. In: Virology. Knipe DM and Howley PM (eds). Lippincott/Williams \& Wilkins Publ Corp., Fields Philadelphia, pp2575-2627, 2001

35. Wilson JB, Bell JL and Levine AJ: Expression of Epstein-Barr virus nuclear antigen-1 induces B cell neoplasia in transgenic mice. EMBO J 15: 3117-3126, 1996.

36. Sheu LF, Chen A, Meng CL, Ho KC, Lee WH, Leu FJ and Chao CF: Enhanced malignant progression of nasopharyngeal carcinoma cells mediated by the expression of Epstein-Barr nuclear antigen 1 in vivo. J Pathol 180: 243-248, 1996.

37. Dolcetti R and Masucci MG: Epstein-Barr virus: induction and control of cell transformation. J Cell Physiol 196: 207-218, 2003.

38. Kang MS, Lu H, Yasui T, Sharpe A, Warren H, Cahir-McFarland E, Bronson R, Hung SC and Kieff E: Epstein-Barr virus nuclear antigen 1 does not induce lymphoma in transgenic FVB mice. Proc Natl Acad Sci USA 102: 820-825, 2005.

39. Liu JY, Chuang TC, Way TD, Tsai TC, Hu CL, Liu GY, Wang SS, Chung JG and Kao MC: The N-terminal domain of EBNA1 acts as a suppressor of the HER2/neu oncogene. Cancer Lett 273: $273-80,2009$

40. Chuang TC, Lee YJ, Liu JY, Lin YS, Li JW, Wang V, Law SL and Kao MC: EBNA1 may prolong G(2)/M phase and sensitize HER2/neu-overexpressing ovarian cancer cells to both topoisomerase II-targeting and paclitaxel drugs. Biochem Biophys Res Commun 307: 653-659, 2003.

41. Jones RJ, Smith LJ, Dawson CW, Haigh T, Blake NW and Young LS: Epstein-Barr virus nuclear antigen 1 (EBNA1) induced cytotoxicity in epithelial cells is associated with EBNA1 degradation and processing. Virology 313: 663-676, 2003.

42. Sheu LF, Chen A, Wei YH, Ho KC, Cheng JY, Meng CL and Lee WH: Epstein-Barr virus LMP1 modulates the malignant potential of gastric carcinoma cells involving apoptosis. Am J Pathol 152: 63-74, 1998.

43. Bertram JS and Janik P: Establishment of a cloned line of Lewis Lung Carcinoma cells adapted to cell culture. Cancer Lett 11: 63-73, 1980.

44. Sheu LF, Lee WC, Lee HS, Kao WY and Chen A: Co-expression of c-kit and stem cell factor in primary and metastatic nasopharyngeal carcinomas and nasopharyngeal epithelium. J Pathol 207: 216-223, 2005.

45. Plumb JA, Milroy R and Kaye SB: Effects of the $\mathrm{pH}$ dependence of 3-(4,5-dimethylthiazol-2-yl)-2,5-diphenyl-tetrazolium bromide-formazan absorption on chemosensitivity determined by a novel tetrazolium-based assay. Cancer Res 49: 4435-4440, 1989.

46. Gupta V, Krishan A and Zubrod CG: Correlation of in vitro clonogenic assay data with in vivo growth delays and cell cycle changes of a human melanoma xenograft. Cancer Res 43: 2560-2564, 1983 .

47. Hakkarainen J, Welsh JA and Vahakangas HK: TP53 mutation detection by SSCP and sequencing. In: Molecular Diagnosis of Cancer. Roulston JE and Bartlett JMS (eds). Humana Press Inc., New Jersey, pp191-208, 2004.
48. Siddik ZH: Cisplatin: mode of cytotoxic action and molecular basis of resistance. Oncogene 22: 7265-7279, 2003.

49. Wood VHJ, O'Neil JD, Wei W, Stewart SE, Dawson CW and Young LS: Epstein-Barr virus-encoded EBNA1 regulates cellular gene transcription and modulates the STAT1 and TGFbeta signaling pathways. Oncogene 26: 4135-4147, 2007.

50. Flavell JR, Baumforth KR, Wood VH, Davies GL, Wei W, Reynolds GM, Morgan S, Boyce A, Kelly GL, Young LS and Murray PG: Down-regulation of the TGF-beta target gene, PTPRK, by the Epstein-Barr virus encoded EBNA1 contributes to the growth and survival of Hodgkin lymphoma cells. Blood 111: 292-301, 2008.

51. Holowaty MN, Sheng Y, Nguyen T, Arrowsmith C and Frappier L: Protein interaction domains of the ubiquitin-specific protease, USP7/HAUSP. J Biol Chem 278: 47753-47761, 2003.

52. Saridakis V, Sheng Y, Sarkari F, Holowaty MN, Shire K., Nguyen T, Zhang RG, Liao J, Lee W, Edwards AM, Arrowsmith $\mathrm{CH}$ and Frappier L: Structure of the p53 binding domain of HAUSP/USP7 bound to Epstein-Barr nuclear antigen 1 implications for EBV-mediated immortalization. Mol Cell 18: 25-36, 2005

53. Joosten SA, Sijpkens YW, van Kooten C and Paul LC: Chronic renal allograft rejection: pathophysiologic considerations. Kidney Int 68: 1-13, 2005.

54. Mocellin S, Wang E, Panelli M, Pilati P and Marincola FM: DNA array-based gene profiling in tumor immunology. Clin Cancer Res 10: 4597-4606, 2004.

55. Marincola FM, Jaffee EM, Hicklin DJ and Ferrone S: Escape of human solid tumors from T-cell recognition: molecular mechanisms and functional significance. Adv Immunol 74: 181-273, 2000.

56. Walker PR, Saas P and Dietrich PY: Role of Fas ligand (CD95L) in immune escape: the tumor cell strikes back. J Immunol 158: 4521-4524, 1997.

57. Drotar M.E, Silva S, Barone E, Campbell D, Tsimbouri P, Jurvansu J, Bhatia P, Klein G and Wilson JB: Epstein-Barr virus nuclear antigen-1 and Myc cooperate in lymphomagenesis. Int J Cancer 106: 388-395, 2003.

58. Zhang M and Coffino P: Repeat sequence of Epstein-Barr virusencoded nuclear antigen 1 protein interrupts proteasome substrate processing. J Biol Chem 279: 8635-8641, 2004.

59. Yin Y, Manoury B and Fahraeus R: Self-inhibition of synthesis and antigen presentation by Epstein-Barr virus-encoded EBNA1. Science 301: 1371-1374, 2003.

60. Munz C: Epstein-Barr virus nuclear antigen 1: from immunologically invisible to a promising T cell target. J Exp Med 199: 1301-1304, 2004.

61. Mautner J, Pich D, Nimmerjahn F, Milosevic S, Adhikary D, Christoph H, Witter K, Bornkamm GW, Hammerschmidt W and Behrends U: Epstein-Barr virus nuclear antigen 1 evades direct immune recognition by $\mathrm{CD}^{+} \mathrm{T}$ helper cells. Eur J Immunol 34: 2500-2509, 2004.

62. Masucci MG: Epstein-Barr virus oncogenesis and the ubiquitinproteasome system. Oncogene 23: 2107-2115, 2004.

63. Murakami M, Lan K, Subramanian C and Robertson ES: Epstein-Barr virus nuclear antigen 1 interacts with $\mathrm{Nm} 23-\mathrm{H} 1$ in lymphoblastoid cell lines and inhibits its ability to suppress cell migration. J Virol 79: 1559-1568, 2005.

64. O'Neil JD, Owen TJ, Wood VH, Date KL, Valentine R, Chukwuma MB, Arrand JR, Dawson CW and Young LS: Epstein-Barr virus-encoded EBNA1 modulates the AP-1 transcription factor pathway in nasopharyngeal carcinoma cells and enhances angiogenesis in vitro. J Gen Virol 89: 2833-2842, 2008.

65. Gruhne B, Sompallae R, Marescotti D, Kamranvar SA, Gastaldello S and Masucci MG: The Epstein-Barr virus nuclear antigen-1 promotes genomic instability via induction of reactive oxygen species. Proc Natl Acad Sci USA 106: 2313-2318, 2009 .

66. Li M, Brooks CL, Kon N and Gu W: A dynamic role of HAUSP in the p53-Mdm2 pathway. Mol Cell 13: 879-886, 2004

67. Holowaty MN and Frappier L: HAUSP/USP7 as an EpsteinBarr virus target. Biochem Soc Trans 32: 731-732, 2004.

68. Ojima H, Fukuda T, Nakajima T and Nagamachi Y: Infrequent overexpression of p53 protein in Epstein-Barr virus-associated gastric carcinomas. Jpn J Cancer Res 88: 262-266, 1997.

69. Hsieh LL, Lin PJ, Chen TC and Ou JT: Frequency of EpsteinBarr virus-associated gastric adenocarcinoma in Taiwan. Cancer Lett 129: 125-129, 1998. 